\title{
Central and peripheral intraocular pressure measured by a rebound tonometer
}

This article was published in the following Dove Press journal:

Clinical Ophthalmology

9 August 201 I

Number of times this article has been viewed

\author{
Tsutomu Yamashita' \\ Atsushi Miki ${ }^{1,2,3}$ \\ Yoshiaki leki $^{2}$ \\ Junichi Kiryu² \\ Kiyoshi Yaoeda ${ }^{3,4}$ \\ Motohiro Shirakashi, ${ }^{3,5}$ \\ 'Department of Sensory Science, \\ Faculty of Health Science and \\ Technology, Kawasaki University \\ of Medical Welfare, ${ }^{2}$ Department of \\ Ophthalmology, Kawasaki Medical \\ School, Kurashiki, Okayama, Japan; \\ ${ }^{3}$ Division of Ophthalmology and Visual \\ Sciences, Niigata University Graduate \\ School of Medical and Dental \\ Sciences, Niigata, Japan; ${ }^{4}$ Yaoeda Eye \\ Clinic, Nagaoka, Japan; ${ }^{5}$ Kido Eye \\ Clinic, Niigata, Japan
}

Correspondence: Atsushi Miki Department of Ophthalmology, Kawasaki Medical School, 577 Matsushima, Kurashiki, Okayama 701-0192, Japan

$\mathrm{Tel}+8 \mathrm{I} 86462$ I I I I

Fax +8I 864630923

Email amiki@tc5.so-net.ne.jp
Purpose: We investigated at which corneal region the intraocular pressure as measured by the Icare ${ }^{\circledR}$ rebound tonometer (Finland Oy, Espoo, Finland) (hereinafter referred to as IC) was closest to the intraocular pressure as measured by the Goldmann applanation tonometer (hereinafter referred to as GT). We also investigated which parameters would be best for preparing the most suitable model for predicting GT.

Methods: A total of 102 normal eyes in 102 subjects were enrolled. IC measurements were carried out at the central, superior, inferior, temporal, and nasal regions of the cornea (ICC, ICS, ICI, ICT, and ICN, respectively), followed by GT calculations. Differences between GT and IC were analyzed using the Bland-Altman method. Stepwise multiple regression analysis was performed using GT as the objective variable, and age, laterality of eye, spherical equivalent refractive error, corneal radius, axial length, central corneal thickness, GT, ICC, ICS, ICI, ICT, and ICN as the explanatory variables.

Results: IC was higher than GT at all of the corneal regions, but the region with the least bias was ICC, followed by ICT. In the multiple regression analysis, the following prediction formula was calculated: $\mathrm{GT}=(0.445 \times \mathrm{ICC})+(0.198 \times \mathrm{ICN})+3.022$. When ICC was excluded from the explanatory variables, ICT had the highest partial correlation coefficient with GT.

Conclusion: ICC was closest to GT, but GT could be explained better by adding ICN to the prediction model. Moreover, in instances where ICC cannot be calculated or where reliability is clearly poor due to abnormal ocular rigidity, ICT was the closest to GT measured in the central corneal region.

Keywords: IOP, Icare, Goldmann applanation tonometer, ocular rigidity

\section{Introduction}

Glaucoma is a disorder that causes serious visual impairment that leads to blindness if not treated appropriately. ${ }^{1}$ The greatest risk factor associated with the onset and progression of glaucoma is elevation in intraocular pressure (IOP). ${ }^{1}$ Therefore, the accurate measurement of IOP is extremely important for glaucoma management in patients.

The current gold standard for tonometers in ophthalmological practice is the Goldmann applanation tonometer (GAT). ${ }^{2}$ However, "IOP as measured by a GAT" (hereinafter referred to as GT) is affected by ocular rigidity. ${ }^{3}$ Ocular rigidity is defined as the distensibility or resistance to deformation in the ocular coat. ${ }^{3}$ If the ocular rigidity is abnormal due to abnormal corneal thickness, corneal disease, or ocular surgery, GT measurements will be inaccurate. ${ }^{4}$ For example, in cases where abnormal ocular rigidity occurs in association with disease or surgery, it is known that GT in the affected 
area is higher than that in other corneal regions, following corneal calcareous degeneration such as band keratopathy. ${ }^{5}$ On the other hand, GT in the affected area is lower than that in other corneal regions in the case of keratoconus ${ }^{6}$ and laser in situ keratomileusis. ${ }^{7}$

Obtaining accurate GT measurements in eyes with abnormal ocular rigidity is not an easy task. In cases where abnormal ocular rigidity of the central corneal region is encountered, IOP must sometimes be measured in the peripheral corneal regions. However, since the area that applanates cornea is large, $3.06 \mathrm{~mm}$ in the case of using a GAT, the measurement might be affected by the ocular rigidity in the central corneal region in no small way when measuring IOP in the peripheral corneal regions. Moreover, obtaining a measurement may be impossible in cases where the measured region contains corneal scarring or sclera.

Icare $^{\circledR}$ (Finland Oy, Espoo, Finland), a recently developed rebound tonometer, makes it possible to measure IOP without anesthesia, and it is portable due to its small size and light weight. ${ }^{8-10}$ In addition, the diameter of the probe tip that comes into contact with the cornea is extremely small at $1.4 \mathrm{~mm},{ }^{10}$ making it easy to measure IOP in peripheral corneal regions.

In the present study, we investigated at which corneal regions "IOP measurements using the Icare rebound tonometer" (hereinafter referred to as IC) were closest to GT at the central corneal region. We also investigated which parameters would be best for preparing the most suitable model for predicting GT.

\section{Subjects and methods}

A total of 102 eyes in 102 subjects (male/female $=52 / 50$ ) without any abnormality on ophthalmologic examination, other than refractive error, were studied. Informed consent was obtained from all subjects prior to the study. Niigata University Institutional Review Board approved all methodology. Study methods adhered to the provisions of the Declaration of Helsinki guidelines for research involving human participants.

Spherical equivalent refractive error (D) and corneal radius $(\mathrm{mm})$ were calculated using the KR-8100P autorefractometer (Topcon Corporation, Tokyo, Japan).

IC was measured in the central corneal region (ICC) and four other regions (superior [ICS], inferior [ICI], temporal [ICT], and nasal [ICN]) located about $2 \mathrm{~mm}$ from the limbus. Subjects were instructed to look head-on (primary position) for measurement of ICC. For measurement of IC in the peripheral corneal regions on the other hand, subjects were instructed to look contralateral (secondary position) to the measured region. For example, subjects were instructed to look downward when measuring ICS. The peripheral corneal measurements were achieved by having the subjects fixate at the target on the wall. The order of the measurements was random. To investigate the reproducibility of Icare tonometry, an averaged IC (ICC) calculated from six consecutive measurements was obtained three times (from 18 measurements) in the central corneal region. In this study, median ICC was used for all analyses other than calculation of coefficients of variation and intraclass correlation coefficients. In the case of the superior, inferior, temporal and nasal corneal regions, IC was measured only once (from six consecutive measurements) to calculate ICS, ICI, ICT, and ICN, respectively.

GT was then measured in the central corneal region using a GAT (Haag-Streit, Koeniz, Switzerland). Finally, the central corneal thickness and axial length of each study eye were measured using an SP-2000 ultrasound pachymeter (Tomey, Nagoya, Japan) and a UD-6000 ultrasonic A/B scanner biometer (Tomey), respectively.

Coefficients of variation and intraclass correlation coefficients were calculated for the three consecutive measurements of ICC. ICC, ICS, ICI, ICT, and ICN were compared using a mixed model with IC as a fixed factor, and subjects and laterality of eye (right/left $=51 / 51$ ) as random factors. The Bonferroni method was used for multiple comparison adjustments.

Pearson correlation coefficients were calculated, and a correlation matrix was prepared for the following parameters: age, spherical equivalent refractive error, corneal radius, axial length, central corneal thickness, GT, ICC, ICS, ICI, ICT, and ICN. Differences between GT and IC were analyzed using the Bland-Altman method.

To generate a model for predicting GT, multiple regression analysis was performed by the stepwise procedure, using GT as the objective variable, and age, laterality of eye, spherical equivalent refractive error, corneal radius, axial length, central corneal thickness, GT, ICC, ICS, ICI, ICT, and ICN as explanatory variables. In addition, assuming there will be instances where ICC cannot be calculated or where reliability is clearly poor due to abnormal ocular rigidity, multiple regression analysis was also performed without ICC as an explanatory variable. When an explanatory variable with a variance inflation factor exceeding 10 was present, another model was generated without the explanatory variable.

All statistical analyses were performed using SPSS $17.0 \mathrm{~J}$ (SPSS Japan Inc, Tokyo, Japan) and MedCalc version 
10.1 (MedCalc Software bvba, Mariakerke, Belgium). A significance level below 5\% was considered to be statistically significant.

\section{Results}

A summary of the 102 eyes in 102 subjects in the present study is shown in Table 1 . The average coefficient of variation of ICC was $8.8 \%$. The intraclass correlation coefficient was 0.879 .

ICS and ICN were significantly higher than ICC ( $P<0.001$ and $P=0.003$, respectively), but there was no difference between any of the other regions. In terms of the correlation matrix of each parameter (Table 2), there was a strong positive correlation between GT and each IC. In addition, there was a significant correlation between the corneal radius and the central corneal thickness, and many ICs.

Differences of GT and IC using the Bland-Altman method are shown in Table 3. ICs were higher than GT in all of the corneal regions, but the IC with the least bias relative to GT was ICC (Figure 1), followed by ICT.

In multiple regression analysis using the stepwise procedure, GT was explained by the following formula:

$$
\begin{aligned}
\mathrm{GT}= & (0.445 \times \mathrm{ICC})+(0.198 \times \mathrm{ICN})+3.022 \\
& \times(\text { coefficient of determination }=0.636)
\end{aligned}
$$

When approximated using only ICC, which had the highest coefficient of determination, GT was explained by the following formula:

$$
\begin{aligned}
\mathrm{GT}= & (0.530 \times \mathrm{ICC})+5.196 \\
& \times(\text { coefficient of determination }=0.599)
\end{aligned}
$$

When ICC was excluded from the explanatory variables, GT was explained by the following formula:

$$
\begin{aligned}
\mathrm{GT}= & (0.203 \times \mathrm{ICT})+(0.159 \times \mathrm{ICS})+(0.215 \times \mathrm{ICN}) \\
& +3.166(\text { coefficient of determination }=0.447)
\end{aligned}
$$

When approximated using only ICT, which had the highest coefficient of determination, GT was explained by the following formula:

$$
\begin{aligned}
\mathrm{GT}= & (0.451 \times \mathrm{ICT})+5.695 \\
& \times(\text { coefficient of determination }=0.360)
\end{aligned}
$$

\section{Discussion}

In the present study, the average of the coefficients of variation of ICC was $8.8 \%$, and the intraclass correlation coefficient was 0.879 . These are comparable to the findings (coefficient of variation: $8.9 \%$, intraclass correlation coefficient: $0.73-0.87$ ) reported from a study by Martinezde-la-Casa et $\mathrm{al}^{11}$ carried out in subjects with glaucoma or ocular hypertension. Therefore, the IC measurements in this investigation have good reproducibility.

In the present study, ICS and ICN were significantly higher than ICC, but there were no differences between any of the other regions. There have been several reports in the past of IOP comparisons in the central and peripheral corneal regions. In a study using a GAT, ${ }^{12}$ there was no difference in GT between the central corneal region and the peripheral

\begin{tabular}{|c|c|c|c|c|}
\hline & Mean & Standard deviation & Minimum & Maximum \\
\hline Age (years) & 36.2 & 8.1 & 21 & 56 \\
\hline Spherical equivalent refractive error (D) & -3.4 & 2.7 & -9.5 & I.I \\
\hline Corneal radius $(\mathrm{mm})$ & 7.9 & 0.3 & 7.2 & 8.6 \\
\hline Axial length (mm) & 25.1 & 1.3 & 22.3 & 28.0 \\
\hline Central corneal thickness $(\mu \mathrm{m})$ & 554.4 & 39.8 & 456.0 & 651.0 \\
\hline $\mathrm{GT}(\mathrm{mmHg})$ & 13.3 & 3.0 & 7 & 21 \\
\hline ICC (mmHg) & 15.3 & 4.4 & 7 & 32 \\
\hline ICS (mmHg) & 18.4 & 5.0 & 9 & 40 \\
\hline $\mathrm{ICl}(\mathrm{mmHg})$ & 17.0 & 4.6 & 9 & 38 \\
\hline ICT (mmHg) & 16.9 & 4.1 & 9 & 29 \\
\hline ICN (mmHg) & 17.6 & 3.6 & II & 28 \\
\hline
\end{tabular}
corneal regions. In studies using the Tono-Pen tonometer, no difference in IOP was reported between the central corneal region and peripheral corneal regions in one study, ${ }^{13}$ while IOP was slightly higher in the temporal corneal region as compared with the central corneal region in another study. ${ }^{14}$ A past study using Icare reported no difference in IOP between the central

Table I Summary of subjects $(n=102$, male/female $=52 / 50$, right eye/left eye $=5 \mathrm{I} / 5 \mathrm{I})$

Abbreviations: GT, intraocular pressure measured by a Goldmann applanation tonometer; ICC, intraocular pressure in the central corneal region measured by Icare ${ }^{\circledR}$; ICS, intraocular pressure in the superior corneal region measured by Icare; ICI, intraocular pressure in the inferior corneal region measured by Icare; ICT, intraocular pressure in the temporal corneal region measured by Icare; ICN, intraocular pressure in the nasal corneal region measured by lcare. 


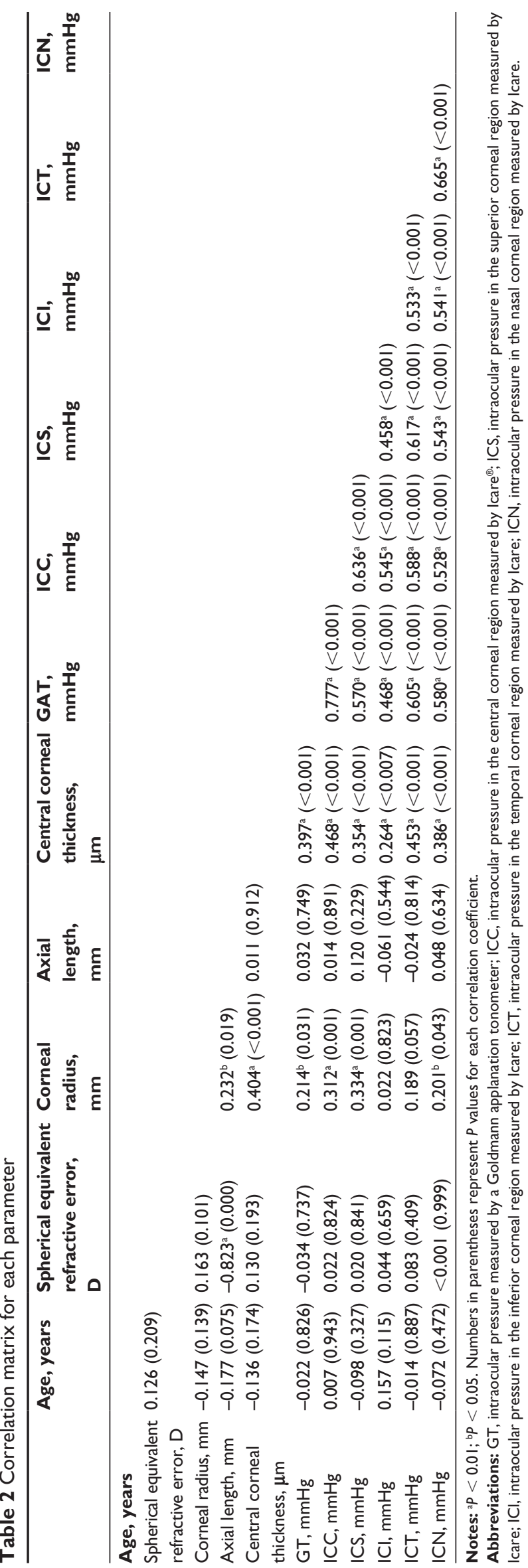

Table 3 Differences in intraocular pressure measured by a Goldmann applanation tonometer and intraocular pressure measured by the Icare ${ }^{\circledR}$ rebound tonometer according to Bland-Altman method

\begin{tabular}{lllll}
\hline & Bias & Precision & $\begin{array}{l}\text { Lower limits } \\
\text { of agreement }\end{array}$ & $\begin{array}{l}\text { Higher limits } \\
\text { of agreement }\end{array}$ \\
\hline GT - ICC & -2.0 & 2.8 & -7.6 & 3.5 \\
GT - ICS & -5.1 & 4.1 & -13.1 & 2.9 \\
GT - ICI & -3.6 & 4.1 & -11.7 & 4.5 \\
GT - ICT & -3.6 & 3.3 & -10.0 & 2.8 \\
GT - ICN & -4.3 & 3.1 & -10.3 & 1.8
\end{tabular}

Abbreviations: GT, intraocular pressure measured by a Goldmann applanation tonometer; ICC, intraocular pressure in the central corneal region measured by Icare; ICS, intraocular pressure in the superior corneal region measured by Icare; $\mathrm{ICl}$, intraocular pressure in the inferior corneal region measured by Icare; ICT, intraocular pressure in the temporal corneal region measured by Icare; ICN, intraocular pressure in the nasal corneal region measured by Icare.

corneal region and peripheral corneal regions, ${ }^{15}$ while ICT was slightly higher than ICC and ICN in another study. ${ }^{16}$ Thus, with respect to differences in IOP between the central and peripheral corneal regions, the results differ depending on the report. In general, the cornea is thicker in the periphery when compared with the central region. ${ }^{16,17}$ Therefore, IOP is assumed to be higher in the peripheral corneal regions when compared to the central corneal region. ${ }^{14}$ Histologically, however, the density of collagen fibers in the peripheral corneal regions is lower than that in the central region, ${ }^{17}$ making it susceptible to corneal tensility and elasticity during the measurement of IOP. ${ }^{18}$ Therefore, IOP measurements may be underestimated in the peripheral corneal regions. Moreover, it has been reported that IOP tends to be higher in the secondary position compared with the primary position. ${ }^{19} \mathrm{~A}$ combination of several of these factors may have led to the discrepancy between these reports. In addition, many of these reports were of studies that used one-way analysis of variance for the statistical examination, but in the present investigation a mixed model was prepared

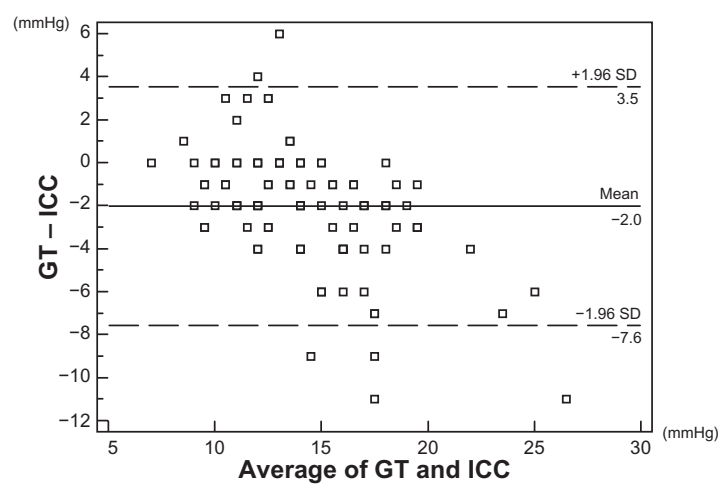

Figure I Scatter diagram of means and differences between GT and ICC according to the Bland-Altman method.

Abbreviations: GT, intraocular pressure measured by a Goldmann applanation tonometer; ICC, intraocular pressure in central corneal region measured using Icare ${ }^{\oplus ;}$ SD, standard deviation. 
using subjects' right and left eyes as random effects, thereby taking into account the effects of the differences between the right and left eyes on IC for comparison of IOP levels between corneal regions in the same cornea.

In the present study, there was a strong positive correlation between GT and IC, with the correlation between GT and ICC being the highest $(r=0.777)$. In a past report involving healthy subjects, ${ }^{10}$ the correlation coefficient between GT and ICC was 0.82. In a report involving patients with glaucoma or ocular hypertension, ${ }^{11}$ the correlation coefficient between GT and ICC was 0.865 . The results of the present study are comparable to the results in these reports. In the present study, differences between GT and IC were investigated using the Bland-Altman method, which showed that the bias between GT and ICC was $-2.0 \mathrm{mmHg}$, while that between GT and IC in the other corneal regions was -3.6 to $-5.1 \mathrm{mmHg}$. Overall, these data indicate that IC was higher than GT at all corneal regions. These results are also comparable to a past report of healthy persons (bias $(\mathrm{GT}-\mathrm{ICC})=-1.94)^{15}$ and the results of a study involving patients with glaucoma or ocular hypertension $($ bias $(\mathrm{GT}-\mathrm{ICC})=-1.8) .{ }^{11}$ Based on these results, we concluded that IC correlates well with GT. However, since some differences were seen, some correction was needed to predict GT based on IC.

In the present study, generation of a model for predicting GT using the stepwise procedure, with each IC as well as age, laterality of eye, spherical equivalent refractive error, corneal radius, axial length, and central corneal thickness as explanatory variables, yielded Equation (1) above. ICC had the highest coefficient of determination with GT $(=0.599)$ and was selected as a significant explanatory variable. Moreover, the coefficient of determination did not decrease much, even when a model was generated with only ICC as an explanatory variable. Although the partial correlation coefficient was low, ICN was also selected as a significant explanatory variable, likely because a GAT was affected more by ocular rigidity in the peripheral cornea compared with Icare, due to the larger corneal contact area during the measurement of IOP. Based on these results, it may be useful to also measure ICN, as well as the ICC, when predicting GT based on IC.

On the other hand, assuming there will be instances where ICC cannot be calculated or where reliability is clearly poor due to abnormal ocular rigidity, multiple regression analysis was also performed without ICC as an explanatory variable. The result was Equation (3). Here, ICT had the highest coefficient of determination $(=0.360)$ and was selected as a significant explanatory variable. Moreover, the coefficient of determination did not decrease much even when a model was generated with only ICT as an explanatory variable. Additionally, when we consider the results of the investigation of the differences between GT and IC yielded by the Bland-Altman method, IOP measurements using Icare in the temporal corneal region might be most useful from the standpoint of predicting GT, since bias was the smallest for ICT among IC in the peripheral corneal regions. However, the coefficient of determination is low compared with a GT prediction model that includes ICC as an explanatory variable, and the difference between GT and ICT according to the Bland-Altman method is also larger than that between GT and ICC. Therefore, it is likely inferior to ICC measurements when trying to obtain consistency with GT measurements.

Parameters thought to be related to ocular rigidity such as corneal curvature radius and central corneal thickness were not selected as significant explanatory variables in these prediction models. According to past reports, ${ }^{4,11}$ corneal curvature radius and central corneal thickness were found to have a significant correlation with GT and ICC, and corneal curvature radius and central corneal thickness were found to have a significant correlation with GT and many ICs in the present study, as well. When generating a multiple regression model using the stepwise procedure, therefore, corneal curvature radius and central corneal thickness were believed to have acted as confounding factors with respect to the objective variable GT and the explanatory variable IC and been removed from the prediction model. Moreover, this revealed that corneal curvature radius and central corneal thickness data are not necessarily required when predicting GT with IC.

IOP decreases when it is measured consecutively with various tonometers, as in the case of IOP measurements by GAT, a phenomenon that was first pointed out during the development of the tonometer. ${ }^{2,20}$ Significant IOP-lowering effects as a result of consecutive measurements are not seen with Icare, or are less than those associated with consecutive measurements using a GAT. ${ }^{21}$ In the present study, IOP was measured with Icare before measurement with GAT, in order to partially prevent the IOP-lowering effects associated with consecutive measurements. However, it was reported that an IOP-lowering effect was seen with consecutive measurement of IOP with a rebound tonometer at the animal experiment level. ${ }^{22}$ In the present study, IOP was measured randomly in each of the corneal regions using Icare, but we cannot rule out the effects of changes in IOP that may have been caused by consecutive measurements.

In conclusion, of the ICs measured in the central or peripheral corneal regions, ICC was the closest to GT. In addition to ICC, GT could be explained better by generating 
a prediction model using ICN. Moreover, in instances where ICC cannot be calculated or where reliability is clearly poor due to abnormal ocular rigidity, ICT was the closest to GT measured in the central corneal region.

\section{Disclosure}

The authors report no conflicts of interest in this work.

\section{References}

1. Japan Glaucoma Society. The Japan glaucoma society guidelines for glaucoma (2nd edition). Nippon Ganka Gakkai Zasshi. 2006;110: 778-814.

2. Goldmann H, Schmidt T. Uber Applanations - Tonometrie. Ophthalmologica. 1957;134:221-242.

3. Damji KF, Muni RH, Munger RM. Influence of corneal variables on accuracy of intraocular pressure measurement. J Glaucoma. 2003;12:69-80.

4. Doughty MJ, Zaman ML. Human corneal thickness and its impact on intraocular pressure measures: a review and meta-analysis approach. Surv Ophthalmol. 2000;44:367-408.

5. Azuara-Blanco A, Bhojani TK, Sarhan AR, Pillai CT, Dua HS. Tono-Pen determination of intraocular pressure in patients with band keratopathy or glued cornea. Br J Ophthalmol. 1998;82:634-636.

6. Böhm A, Kohlhaas M, Lerche RC, Bischoff B, Richard G. Measuring intraocular pressure in keratoconus. Effect of the changed biomechanics. Ophthalmologe. 1997;94:771-774.

7. Emara B, Probst LE, Tingey DP, et al. Correlation of intraocular pressure and central corneal thickness in normal myopic eyes and after laser in situ keratomileusis. J Cataract Refract Surg. 1998;24:1320-1325.

8. Kontiola A. A new electromechanical method for measuring intraocular pressure. Doc Ophthalmol. 1996-1997;93:265-276.

9. Kontiola A, Puska P. Measuring intraocular pressure with the Pulsair 3000 and Rebound tonometers in elderly patients without an anesthetic. Graefes Arch Clin Exp Ophthalmol. 2004;242:3-7.
10. Kontiola AI. A new induction-based impact method for measuring intraocular pressure. Acta Ophthalmol Scand. 2000;78:142-145.

11. Martinez-de-la-Casa JM, Garcia-Feijoo J, Castillo A, Garcia-Sanchez J. Reproducibility and clinical evaluation of rebound tonometry. Invest Ophthalmol Vis Sci. 2005;46:4578-4580.

12. Whitacre MM, Stein R. Sources of error with use of Goldmann-type tonometers. Surv Ophthalmol. 1993;38:1-30.

13. Mok KH, Wong CS, Lee VW. Tono-Pen tonometer and corneal thickness. Eye. 1999;13:35-37.

14. Khan JA, Davis M, Graham CE, Trank J, Whitacre MM. Comparison of Oculab Tono-Pen readings obtained from various corneal and scleral locations. Arch Ophthalmol. 1991;109:1444-1446.

15. Chui WS, Lam A, Chen D, Chiu R. The influence of corneal properties on rebound tonometry. Ophthalmology. 2008,115:80-84.

16. Jorge JM, González-Méijome JM, Queirós A, Fernandes P, Parafita MA. Correlations between corneal biomechanical properties measured with the ocular response analyzer and ICare rebound tonometry. J Glaucoma. 2008; 17:442-448

17. Boote C, Dennis S, Newton RH, Puri H, Meek KM. Collagen fibrils appear more closely packed in the prepupillary cornea: optical and biomechanical implications. Invest Ophthalmol Vis Sci. 2003;44:2941-2948.

18. Herndon LW. Measuring intraocular pressure - adjustments for corneal thickness and new technologies. Curr Opin Ophthalmol. 2006;17:115-119.

19. Nardi M, Bartolomei MP, Romani A, Barca L. Intraocular pressure changes in secondary positions of gaze in normal subjects and in restrictive ocular motility disorders. Graefes Arch Clin Exp Ophthalmol. 1988;226:8-10.

20. Krakau CE, Wilke K. On repeated tonometry. Acta Ophthalmol (Copenh). 1971;49:611-614.

21. Davies LN, Bartlett H, Mallen EA, Wolffsohn JS. Clinical evaluation of rebound tonometer. Acta Ophthalmol Scand. 2006;84:206-209.

22. Morris CA, Crowston JG, Lindsey JD, Danias J, Weinreb RN. Comparison of invasive and non-invasive tonometry in the mouse. Exp Eye Res. 2006;82:1094-1099.
Clinical Ophthalmology

\section{Publish your work in this journal}

Clinical Ophthalmology is an international, peer-reviewed journal covering all subspecialties within ophthalmology. Key topics include: Optometry; Visual science; Pharmacology and drug therapy in eye diseases; Basic Sciences; Primary and Secondary eye care; Patient Safety and Quality of Care Improvements. This journal is indexed on Submit your manuscript here: http://www.dovepress.com/clinical-ophthalmology-journal

\section{Dovepress}

PubMed Central and CAS, and is the official journal of The Society of Clinical Ophthalmology (SCO). The manuscript management system is completely online and includes a very quick and fair peer-review system, which is all easy to use. Visit http://www.dovepress.com/ testimonials.php to read real quotes from published authors. 